\title{
Bayesian Bonus-Malus Premium with Poisson-Lindley Distributed Claim Frequency and Lognormal-Gamma Distributed Claim Severity in Automobile Insurance
}

\author{
ADISAK MOUMEESRI ${ }^{1}$, WATCHARIN KLONGDEE ${ }^{2}$, TIPPATAI PONGSART ${ }^{3 *}$ \\ ${ }^{1,2}$ Department of Mathematics, Faculty of Science, Khon Kaen University, Khon Kaen, THAILAND \\ ${ }^{3}$ Department of Statistics, Faculty of Science, Khon Kaen University, Khon Kaen, THAILAND
}

\begin{abstract}
The traditional automobile insurance bonus-malus system (BMS) merit-rating depends on the number of claims. An insured individual who makes a small severity claim is penalized unfairly compared to an insured person who makes a large severity claim. A model for assigning the bonus-malus premium was proposed. Consideration was based on both the number and size of the claims that were assumed to follow a Poisson-Lindley distribution and a Lognormal-Gamma distribution, respectively. The Bayesian method was applied to compute the bonus-malus premiums, integrated by both frequency and severity components based on the posterior criteria. Practical examples using a real data set are provided. This approach offers a fairer method of penalizing all policyholders in the portfolio.
\end{abstract}

Key-Words: - Automobile insurance, Bayesian method, Bonus-malus system, Claim severity, Number of claims, Poisson-Lindley distribution, Lognormal-Gamma distribution

Received: April 1, 2020. Revised: August 19, 2020. Accepted: August 29, 2020. Published: September 20, 2020.

\section{Introduction}

Automobile insurance is an essential branch of nonlife insurance. A bonus-malus system (BMS) is widely used in compulsory automobile liability insurance to adjust premiums paid by policyholders based on their particular claim history. A bonus as a discount is offered as a reward for accident-free driving, while a malus results in an increase in the premium. BMS premium pricing initially depends on the recorded numbers of claims in previous years. If the policyholder makes a claim, he or she will be charged a higher premium (malus) for the next insurance period. On the other hand, if no claim is made, a lower premium (bonus) will be charged for the renewal. The main goal of this system is to reward good drivers and penalize bad drivers. Insurance companies take advantage of the BMS for two main reasons. The first is to encourage policyholders to drive carefully during the year and make no claims, while the second is to ensure that policyholders pay premiums proportional to their risk value based on previous claims.

The Bayesian method is a tool to be very useful for calculating premiums. The method accepts each policyholder has a constant as an unequal underlying risk. This constant is called a risk parameter which is unknown and is treated as a random variable with a particular probability distribution. The distribution is called a prior distribution or structure-function. This approach is convenient to compute premiums in the BMS since the premiums are based on the specific transition rules which distinguish the policyholders as a bonus or malus. The basic Bayesian tool is easily applied to Bayes' theorem by dividing a posterior mean of the parameter considering a prior mean. The net premium principle is utilized in this tool. This will provide an estimate of the risk parameter for separating the policyholders between good and bad risks.

The Poisson distribution is usually used for the explanation of independent and random events. It is applied to many studies, including a traffic modeling, see also [1], and a tracking area planning approach, see even [2]. In addition, it is used for the description of the random event of claims in automobile insurance. The Poisson distribution can be used to express the probability of the behavior of individual policyholders. However, it cannot adequately describe the number of claims in an insurance portfolio. A mixed Poisson was proposed for claim frequency distribution by [3] who designed an optimal BMS by mixing the Poisson distribution with the Inverse Gaussian distribution for claim frequency. A random number of claims that varied with the portfolio was also assumed to be Poisson distributed, while the randomly expected inherent risks of each insured person followed 
inverse Gaussian distribution. Here, Bayesian methods were applied to estimate the posterior portfolio distribution function for a scenario covering the past $t$ years. A fair premium was determined using the zero-utility principle. Reference [4] introduced an optimal BMS considering the number of claims in different distributions. Mixing Poisson with Gamma distributions was considered for the Negative Binomial claim frequency distribution. The expected premium value was calculated based on the BMS principle. Reference [5] extended the methods of [3] and [4] who used the three-parameter Hofmann's distribution and showed that this gave a better fit for the claim frequency data. Several papers have discussed mixing other distributions to obtain an optimal premium for the number of claims. See, for example, [6]-[8].

Our analysis suggested that previous evaluations showed no difference in payment between a claim made by a policyholder for a small loss and another with a big loss. It seems unfair to penalize all policyholders equally. For instance, a claim of US $\$ 50$ by a policyholder should not be penalized by the same increase in premium as a claim of US\$500. Therefore, when assigning the insurance premium to be charged, more factors must be taken into account than considering only a model frequency component. To resolve this discrepancy, an optimal BMS was developed by (Reference [9]) taking both the frequency and severity component into account, followed by Poisson-Gamma distribution and Exponential-Inverse Gamma (Pareto distribution), respectively. In particular, the number of claims was assumed to be Poisson distributed with mean $\lambda$, where $\lambda$ is the underlying risk that varies depending on each policyholder. The underlying risk was assumed to be Gamma distributed as a random variable. For the severity component, the claim amount was assumed to be an Exponential distribution, while its mean was assumed to be Inverse Gamma distributed. Using the Bayesian method, the posterior structure functions of the frequency component and the severity component were obtained for the number of years that the policyholder had been under observation. The premium estimate was based on the net premium principle, as a product of the mean of the posterior distribution function for the frequency component and the posterior distribution function for the severity component. In particular, the suitable premiums based on BMS were derived using the following multiplicative formula:

Premium $=\mathrm{E}[$ Frequency component $] \times \mathrm{E}[$ Severity component $]$
The useful multiplicative formula proposed in (1) was interested in many researchers with considering different distributions in frequency and severity components. Reference [10] considered both frequency and severity components in the design of an optimal BMS by assuming that claim frequency had a Geometric distribution (mixed Poison with Exponential distribution), and claim severity was Pareto distributed. Reference [11] considered the design of optimal BMS based on both frequency and severity components using mixed Poisson with Exponential distribution and mixed Poisson with Gamma for the frequency component. The number of claims was assumed to be Poisson distributed, while the underlying risk of each policyholder was taken to be Exponential and Gamma distributed called the prior distribution. Claim size was modeled as a Pareto distribution, where the claim size for the $k^{\text {th }}$ claim was assumed to be exponentially distributed, and the mean claim amount was assumed to be Inverse Gamma distributed. Reference [12] assumed a Negative Binomial distribution for the frequency component. For the severity component, they focused on modeling claim severity as a Weibull distribution by mixing an Exponential with a Levy distribution. Results for these models were compared for Weibull severity and Pareto severity. Reference [13] allowed Negative Binomial distribution for the frequency component while mixing the Gamma with Gamma distribution was allowed for the severity component. The Bayesian method was then applied to derive the premium based on the BMS. Reference [14] considered the design of an optimal BMS based on both frequency and severity components. They proposed the Exponential-Lognormal (ELN) regression model for severity distribution as a competitive alternative to the Pareto. The number of claims was assumed to be classically Negative Binomial distributed and Poisson-Inverse Gaussian distributed. Reference [15] considered the design of an alternative optimal BMS by assuming that the distribution of claim frequency and severity had Negative Binomial and Pareto, respectively. The net premium principle was considered to calculate the premiums.

Here, we propose a new claim frequency distribution and claim severity distribution to determine optimal premium based on the BMS. The number of claims is considered when mixing the Poisson with the Lindley distribution to assess the claim frequency distribution. The Lindley distribution usually has a thicker tail than an Exponential distribution; therefore, it should provide a better fit to the claim data set. To increase clarity, 
we employed the Poisson-Lindley distribution for claim frequency distribution, while fitting values were compared using the real data set. For claim severity distribution, claim size in automobile insurance is usually heavy tail distributed. Many previous papers used the Pareto distribution to model claim severity. However, Pareto distribution does not adequately fit the claim data. Other heavy tail distributions, for example, Gamma and Lognormal are appropriate to model claim severity distribution, see also [16]-[18]. In this study, we propose the Lognormal-Gamma to model the claim severity distribution. The Bayesian method is applied to obtain the posterior structure functions for claim frequency and claim severity distributions. The mean of these functions is used to estimate the premiums to be charged to a policyholder who has been under observation. Suitable premiums based on BMS were derived using the multiplicative formula shown in (1).

The remainder of this paper is organized as follows. Section 2 describes the research methodology separated into two subsections of claim frequency distribution and claim severity distribution. Mixing distributions, parameter estimations, the goodness of fit test, the Bayesian method, and the premium calculation are also explained. In Section 3, the numerical application is illustrated and results are discussed using the real claim data separated as claim frequency components and claim severity components. Finally, conclusions are drawn and presented in Section 4.

\section{Research Methodology}

We assumed that the frequency and severity of the claim of each policyholder were independent. The claim frequency and claim severity distributions were separated into the following subsections.

\subsection{Claim Frequency Distribution using Poisson-Lindley}

In automobile insurance, all policyholders have a constant as an unequal underlying risk that an accident will occur. This constant is called the risk parameter and is treated as a random variable that depends on each policyholder. The distribution is called the prior distribution. Mixing the Poisson distribution with the prior distributions gives thicker tails than only the Poisson distribution, hence the mixed Poisson distribution compared with the Poisson distribution provides a better fit to the claim frequency data. Thus, we proposed mixing Poisson distribution with Lindley distribution to model the frequency distribution.

\subsubsection{Mixing Distribution}

Assume that the number of claims $k$ is distributed according to the Poisson given parameter $\theta$ with probability mass function

$$
f(k \mid \theta)=\frac{e^{-\theta} \theta^{k}}{k !}, k=0,1,2, \ldots, \theta>0 .
$$

The expected value of the Poisson random variable is $E[K \mid \theta]=\theta$.

All policyholders have a constant representing the expected inherent risk of each insured. This is the mean of the number of claims of each insured, denoted by $\theta$. Assume that $\theta$ is distributed according to the Lindley distribution with parameter $\delta$ (that is, the structure-function of $\theta$ is assumed to be Lindley distribution). Then, the probability density function (pdf) of $\theta$ can be represented as the following form:

$$
\pi(\theta)=\frac{\delta^{2}}{\delta+1}(\theta+1) e^{-\delta \theta}, \theta>0, \delta>0 .
$$

The mixed Poisson with Lindley distribution is obtained as detailed below:

$$
\begin{aligned}
f(k) & =\int_{0}^{\infty} f(k \mid \theta) \pi(\theta) d \theta \\
& =\int_{0}^{\infty} \frac{e^{-\theta} \theta^{k}}{k !} \cdot \frac{\delta^{2}}{\delta+1}(\theta+1) e^{-\delta \theta} d \theta \\
& =\frac{\delta^{2}(k+\delta+2)}{(\delta+1)^{k+3}},
\end{aligned}
$$

where $k=0,1,2, \ldots$ and $\delta>0$.

\subsubsection{Parameter Estimation}

The maximum likelihood estimation (MLE) is widely used to estimate model parameters. The basic idea is to choose a parameter value that maximizes the likelihood function, which is the probability density of the observed data. The principle of maximum likelihood is to estimate the value of parameters that make the observed data most probable. The methodology of MLE for Poisson-Lindley distribution is presented as follows:

Let $k_{1}, k_{2}, \ldots, k_{n}$ be a random sample of size $n$ from Poisson-Lindley distribution with pdf in (2). To find the most likely value of the parameter $\delta$ requires maximizing the likelihood function $L$ where

$$
L\left(\delta ; k_{i}\right)=\prod_{i=1}^{n} f\left(k_{i} ; \delta\right)=\prod_{i=1}^{n} \frac{\delta^{2}\left(k_{i}+\delta+2\right)}{(\delta+1)^{k_{i}+3}} .
$$

Then, the log-likelihood function is

$\ln L\left(\delta ; k_{i}\right)=\sum_{i=1}^{n} \ln \left[\frac{\delta^{2}\left(k_{i}+\delta+2\right)}{(\delta+1)^{k_{i}+3}}\right]$ 


$$
\begin{array}{r}
=2 n \ln \delta+\sum_{i=1}^{n} \ln \left(k_{i}+\delta+2\right) \\
-\ln (\delta+1) \sum_{i=1}^{n}\left(k_{i}+3\right) .
\end{array}
$$

The estimator $\hat{\delta}$ of the parameter $\delta$ can be obtained by solving the equation:

We have

$$
\frac{d}{d \delta} \ln L\left(\delta ; k_{i}\right)=0 .
$$

Thus

$$
\frac{d}{d \delta}\left(2 n \ln \delta+\sum_{i=1}^{n} \ln \left(k_{i}+\delta+2\right)-\ln (\delta+1) \sum_{i=1}^{n}\left(k_{i}+3\right)\right)=0 .
$$

$$
\frac{2 n}{\delta}+\sum_{i=1}^{n}\left(\frac{1}{k_{i}+\delta+2}\right)-\frac{1}{\delta+1} \sum_{i=1}^{n}\left(k_{i}+3\right)=0 .
$$

Since the estimation of the parameters $\delta$ cannot be found in closed form, the numerical iteration technique, bisection method, is used to solve (3).

\subsubsection{Goodness of Fit Test}

For the frequency component, the Chi-Square goodness of fit test was used to describe how the observation of a given phenomenon is significantly different from the expected value. Chi-Square is used to compare the observed sample distribution with the expected probability distribution, and how well the theoretical distribution is suited to the empirical distribution. The sample data were divided into intervals. Then the numbers of points that fell into each interval were compared with the expected number of points in each interval. The value of the Chi-Square goodness of fit test was computed using the following formula:

$$
\chi^{2}=\sum_{i=1}^{n} \frac{\left(O_{i}-E_{i}\right)^{2}}{E_{i}}
$$

where $\chi^{2}$ is the value of the Chi-Square goodness of fit test, $O_{i}$ is the observed frequency count for the $i^{\text {th }}$ level of the categorical variable and $E_{i}$ is the expected frequency count for the $i^{\text {th }}$ level of the categorical variable.

\subsubsection{Bayesian Method}

The bonus-malus premium calculation has been studied using several methods, with Bayesian methodology as one of the most popular methods. The main purpose of this approach is to obtain the posterior distribution function. The Bayesian methodology can be applied when the data for each policyholder is available, whether based on claim history for previous periods or policyholder profiles.

Let $k=\left(k_{1}, k_{2}, \ldots, k_{t}\right)$ be a sample, where $t$ is the sample size. Let $N=\sum_{i=1}^{t} k_{i}$ be the total number of claims made by a policyholder over $t$ years, where $k_{i}$ is the number of claims that the policyholder made in years $i, i=1,2, \ldots, t$. The likelihood function is

$$
\begin{aligned}
L\left(\theta ; k_{1}, k_{2}, \ldots, k_{t}\right) & =P\left(k_{1}, k_{2}, \ldots, k_{t} \mid \theta\right) \\
& =\prod_{i=1}^{t} \frac{e^{-\theta} \theta^{k_{i}}}{k_{i} !} \\
& \propto e^{-\theta t} \theta^{N} .
\end{aligned}
$$

The prior distribution is

$$
\pi(\theta) \propto(\theta+1) e^{-\delta \theta}
$$

Then, the posterior distribution function for a policyholder, or a group of policyholders, with claim history $k_{1}, k_{2}, \ldots, k_{t}$ can be obtained by applying Bayes' theorem. The posterior distribution function is proportional to the product of prior distribution and the likelihood function.

$$
\begin{aligned}
\pi^{*}\left(\theta \mid k_{1}, k_{2}, \ldots, k_{t}\right) & \propto P\left(k_{1}, k_{2}, \ldots, k_{t} \mid \theta\right) \pi(\theta) \\
& =e^{-\theta t} \theta^{N}(\theta+1) e^{-\delta \theta} \\
& =e^{-(t+\delta) \theta}(\theta+1) \theta^{N}
\end{aligned}
$$

Consider

$$
\begin{aligned}
& \int_{0}^{\infty} \pi^{*}\left(\theta \mid k_{1}, k_{2}, \ldots, k_{t}\right) d \theta \propto \int_{0}^{\infty} e^{-(t+\delta) \theta}(\theta+1) \theta^{N} d \theta, \\
& \text { then } \\
& \int_{0}^{\infty} \pi^{*}\left(\theta \mid k_{1}, k_{2}, \ldots, k_{t}\right) d \theta=\int_{0}^{\infty} A e^{-(t+\delta) \theta}(\theta+1) \theta^{N} d \theta=1,
\end{aligned}
$$

where $A$ is a constant. It results in

$$
A=\frac{(t+\delta)^{N+2}}{\Gamma(N+1)(N+1+t+\delta)} .
$$

Therefore, the posterior distribution function for the frequency component can be expressed as

$\pi^{*}\left(\theta \mid k_{1}, k_{2}, \ldots, k_{t}\right)=\frac{(t+\delta)^{N+2}}{\Gamma(N+1)(N+1+t+\delta)} e^{-(t+\delta) \theta}(\theta+1) \theta^{N}$.

\subsubsection{Premium Calculation}

Many principles are involved in pricing insurance premiums. In this article, we determine the net premium principle. This is the basic principle in the sense that premiums should be the expected value of losses. The net premium in such a situation is the expected number or mean of the number of claims that occur from each policyholder.

The expected number of claims of a policyholder with a claim history $k_{1}, k_{2}, \ldots, k_{t}$ or the mean of the posterior distribution function from (4) for Poisson-Lindley distribution will be

$$
\begin{aligned}
\hat{\theta}_{t+1} & =E\left[\theta \mid k_{1}, k_{2}, \ldots, k_{t}\right]=E\left[k_{1}, k_{2}, \ldots, k_{t} \mid \theta\right] \\
& =\int_{0}^{\infty} \theta \frac{(t+\delta)^{N+2}}{\Gamma(N+1)(N+1+t+\delta)} e^{-(t+\delta) \theta}(\theta+1) \theta^{N} d \theta \\
& =\frac{(t+\delta)^{N+2}}{\Gamma(N+1)(N+1+t+\delta)}\left[\frac{\Gamma(N+3)}{(t+\delta)^{N+3}}+\frac{\Gamma(N+2)}{(t+\delta)^{N+2}}\right] \\
& =\frac{(N+1)(N+2+t+\delta)}{(t+\delta)(N+1+t+\delta)} .
\end{aligned}
$$


Assume that the initial premium or base premium at time $t=0$ is 100 . Then, at time $t+1$, the premium can be determined from the number of claims and expressed as the following form:

Premium $_{t+1}=100 \cdot \frac{\delta(1+\delta)(N+1)(N+2+t+\delta)}{(2+\delta)(t+\delta)(N+1+t+\delta)}$.

\subsection{Claim Severity Distribution using Lognormal-Gamma}

In an insurance portfolio, heavy tail distributions such as Gamma, Lognormal, Weibull, Pareto, and Burr are commonly used to model claim severity distribution. Since mixing these claim severity distributions with the prior distribution gives thicker tails, the mixed claim severity distribution provides a better fit to the claim severity data.

\subsubsection{Mixing Distribution}

In the literature, mixed Exponential distribution has been widely used to model severity distributions, see also [9]-[12]. In this article, we propose a mixed Lognormal distribution with Gamma prior distribution to model severity distribution as follows:

Let $X$ be a random variable of the claim size of each insured person. Assume that the size of claim $x$ follows a Lognormal distribution. Then, given that parameter $\lambda=\frac{1}{\sigma^{2}}$, the pdf of Lognormal distribution is given by

$$
f(x \mid \lambda)=\frac{\sqrt{\lambda}}{x \sqrt{2 \pi}} e^{-\frac{\lambda}{2}(\ln x-\mu)^{2}}, x>0, \mu>0, \lambda>0 .
$$

The expected value of $X$ will be $E[X \mid \lambda]=e^{\mu+\frac{1}{2 \lambda}}$.

The parameter $\lambda$ has different values according to each policyholder; therefore, it is reasonable to express $\lambda$ as a distribution. The Gamma prior distribution for $\lambda$ with parameters $\alpha$ and $\beta$ has the pdf as the following form:

$$
\pi(\lambda)=\frac{\beta^{\alpha}}{\Gamma(\alpha)} \lambda^{\alpha-1} e^{-\beta \lambda}, \lambda>0, \alpha>0, \beta>0 .
$$

Unconditional distribution of the claim size $x$ can be obtained as following below.

$$
\begin{aligned}
f(x) & =\int_{0}^{\infty} f(x \mid \lambda) \pi(\lambda) d \lambda \\
& =\int_{0}^{\infty} \frac{\sqrt{\lambda}}{x \sqrt{2 \pi}} e^{-\frac{\lambda}{2}(\ln x-\mu)^{2}} \cdot \frac{\beta^{\alpha}}{\Gamma(\alpha)} \lambda^{\alpha-1} e^{-\beta \lambda} d \lambda \\
& =\frac{\beta^{\alpha}}{x \sqrt{2 \pi} \Gamma(\alpha)} \cdot \frac{\Gamma\left(\alpha+\frac{1}{2}\right)}{\left(\beta+\frac{1}{2}(\ln x-\mu)^{2}\right)^{\alpha+\frac{1}{2}}},
\end{aligned}
$$

where $x>0, \alpha>0$, and $\beta>0$.

\subsubsection{Parameter Estimation}

The methodology of the MLE for Lognormal-
Gamma distribution is presented as follows:

Let vector $X=\left(x_{1}, x_{2}, \ldots, x_{n}\right)^{T}$ be an identically independent observation for LognormalGamma distribution with pdf in (7). To find the most likely value of parameters $\alpha$ and $\beta$ that produce outcome $X$, the likelihood function $L$ must be maximized, where

$$
\begin{aligned}
L\left(\alpha, \beta ; x_{i}\right) & =\prod_{i=1}^{n} f\left(x_{i} ; \alpha, \beta\right) \\
& =\prod_{i=1}^{n}\left[\frac{\beta^{\alpha}}{x_{i} \sqrt{2 \pi} \Gamma(\alpha)} \cdot \frac{\Gamma\left(\alpha+\frac{1}{2}\right)}{\left(\beta+\frac{1}{2}\left(\ln x_{i}-\mu\right)^{2}\right)^{\alpha+\frac{1}{2}}}\right] .
\end{aligned}
$$

Then, the log-likelihood function is provided as follow:

$$
\begin{array}{r}
\ln L\left(\alpha, \beta ; x_{i}\right)=\sum_{i=1}^{n} \ln \left[\frac{\beta^{\alpha}}{x_{i} \sqrt{2 \pi} \Gamma(\alpha)} \cdot \frac{\Gamma\left(\alpha+\frac{1}{2}\right)}{\left(\beta+\frac{1}{2}\left(\ln x_{i}-\mu\right)^{2}\right)^{\alpha+\frac{1}{2}}}\right] . \\
=n\left[\alpha \ln \beta+\ln \Gamma\left(\alpha+\frac{1}{2}\right)-\ln \sqrt{2 \pi}-\ln \Gamma(\alpha)\right]-\sum_{i=1}^{n} \ln x_{i} \\
-\left(\alpha+\frac{1}{2}\right) \sum_{i=1}^{n} \ln \left(\beta+\frac{1}{2}\left(\ln x_{i}-\mu\right)^{2}\right) .
\end{array}
$$

The estimators $\hat{\alpha}$ and $\hat{\beta}$ for $\alpha$ and $\beta$ respectively are obtained by

$$
\frac{\partial}{\partial \alpha} \ln L\left(\alpha, \beta ; x_{i}\right)=0 \quad \text { and } \frac{\partial}{\partial \beta} \ln L\left(\alpha, \beta ; x_{i}\right)=0 .
$$

Then

$$
\begin{aligned}
\frac{\partial}{\partial \alpha} \ln L\left(\alpha, \beta ; x_{i}\right) & =n\left[\ln \beta+\frac{\partial}{\partial \alpha} \ln \Gamma\left(\alpha+\frac{1}{2}\right)-\frac{\partial}{\partial \alpha} \Gamma(\alpha)\right] \\
& -\sum_{i=1}^{n} \ln \left(\beta+\frac{1}{2}\left(\ln x_{i}-\mu\right)^{2}\right)=0
\end{aligned}
$$

and

$$
\frac{\partial}{\partial \beta} \ln L\left(\alpha, \beta ; x_{i}\right)=\frac{\alpha n}{\beta}-\left(\alpha+\frac{1}{2}\right) \sum_{i=1}^{n}\left(\frac{1}{\beta+\frac{1}{2}\left(\ln x_{i}-\mu\right)^{2}}\right)=0 .
$$

We have

$$
\alpha=\frac{\frac{\beta}{2} \sum_{i=1}^{n}\left(\frac{1}{\beta+\frac{1}{2}\left(\ln x_{i}-\mu\right)^{2}}\right)}{n-\beta \sum_{i=1}^{n}\left(\frac{1}{\beta+\frac{1}{2}\left(\ln x_{i}-\mu\right)^{2}}\right)} .
$$

Replacing (9) in (8), we get the parameter $\beta$. Since the estimation of the parameter $\beta$ cannot be found in closed form, the numerical iteration technique, bisection method, is used to solve (8).

\subsubsection{Goodness of Fit Test}

Akaike Information Criterion (AIC) was used as the model selection criteria. The minimum AIC illustrated a good model among all the others with a better fit to the claim data. The equation used to estimate the AIC of the model can be represented as

$$
\mathrm{AIC}=-2 \ln (L)+2 m \text {, }
$$


where $L$ is the value of the likelihood function of the model, and $m$ is the number of estimated parameters in the model.

In this study, we used the AIC to measure the relative quality of the statistical models for claim severity distributions.

\subsubsection{Bayesian Method}

The total number of claims that a policyholder made in $t$ years was represented as $N=\sum_{i=1}^{t} k_{i}$. Let $x_{k}$ denote the size of claim $k$ for $k=1,2, \ldots, N$. Then his/her claim size history in $t$ years can be displayed in the form of a vector $x=\left(x_{1}, x_{2}, \ldots, x_{N}\right)$. The total claim size over $t$ years is $\sum_{k=1}^{N} x_{k}$. The likelihood function is

$$
\begin{aligned}
L\left(\lambda ; x_{1}, x_{2}, \ldots, x_{N}\right) & =f\left(x_{1}, x_{2}, \ldots, x_{N} \mid \lambda\right) \\
& =\prod_{k=1}^{N} \frac{\sqrt{\lambda}}{x_{k} \sqrt{2 \pi}} e^{-\frac{\lambda}{2}\left(\ln x_{k}-\mu\right)^{2}} \\
& \propto \lambda^{\frac{N}{2}} e^{-\frac{\lambda}{2} \sum_{k=1}^{N}\left(\ln x_{k}-\mu\right)^{2}} .
\end{aligned}
$$

The prior distribution is

$$
\pi(\lambda) \propto \lambda^{\alpha-1} e^{-\beta \lambda} .
$$

By applying Bayes' theorem, the posterior distribution function is proportional to the product of prior distribution and the likelihood function, that is

$$
\begin{aligned}
\pi^{*}\left(\lambda \mid x_{1}, x_{2}, \ldots, x_{N}\right) & \propto f\left(x_{1}, x_{2}, \ldots, x_{N} \mid \lambda\right) \pi(\lambda) \\
& =e^{-\left(\beta+\frac{1}{2} \Sigma_{k=1}^{N}\left(\ln x_{k}-\mu\right)^{2}\right) \lambda} \lambda^{\frac{N}{2}+\alpha-1} .
\end{aligned}
$$

Consider

$\int_{0}^{\infty} \pi^{*}\left(\lambda \mid x_{1}, x_{2}, \ldots, x_{N}\right) d \lambda \propto \int_{0}^{\infty} e^{-\left(\beta+\frac{1}{2} \sum_{k=1}^{N}\left(\ln x_{k}-\mu\right)^{2}\right) \lambda} \lambda^{\frac{N}{2}+\alpha-1} d \lambda$, then

$\int_{0}^{\infty} \pi^{*}\left(\lambda \mid x_{1}, x_{2}, \ldots, x_{N}\right) d \lambda=\int_{0}^{\infty} B e^{-\left(\beta+\frac{1}{2} \sum_{k=1}^{N}\left(\ln x_{k}-\mu\right)^{2}\right) \lambda} \lambda^{\frac{N}{2}+\alpha-1} d \lambda=1$.

where $B$ is a constant. We get

$$
B=\frac{\left(\beta+\frac{1}{2} \sum_{k=1}^{N}\left(\ln x_{k}-\mu\right)^{2}\right)^{\frac{N}{2}+\alpha}}{\Gamma\left(\frac{N}{2}+\alpha\right)} .
$$

Therefore, the posterior distribution function for severity distribution can be presented in the following form:

$\pi^{*}\left(\lambda \mid x_{1}, x_{2}, \ldots, x_{N}\right)$

$=\frac{\left(\beta+\frac{1}{2} \sum_{k=1}^{N}\left(\ln x_{k}-\mu\right)^{2}\right)^{\frac{N}{2}+\alpha}}{\Gamma\left(\frac{N}{2}+\alpha\right)} e^{-\left(\beta+\frac{1}{2} \sum_{k=1}^{N}\left(\ln x_{k}-\mu\right)^{2}\right) \lambda} \lambda^{\frac{N}{2}+\alpha-1} \cdot(10)$

\subsubsection{Premium Calculation}

For claim severity distribution, we used the net premium principle for computing the premium, similar to the claim frequency distribution. The expected value in (10) of Lognormal-Gamma distribution was

$$
\hat{\lambda}_{t+1}=E\left[\lambda \mid x_{1}, x_{2}, \ldots, x_{N}\right]
$$

$$
\begin{aligned}
& =\int_{0}^{\infty} \lambda \frac{\left(\beta+\frac{1}{2} \sum_{k=1}^{N}\left(\ln x_{k}-\mu\right)^{2}\right)^{\frac{N}{2}+\alpha}}{\Gamma\left(\frac{N}{2}+\alpha\right)} e^{-\left(\beta+\frac{1}{2} \sum_{k=1}^{N}\left(\ln x_{k}-\mu\right)^{2}\right) \lambda} \lambda^{\frac{N}{2}+\alpha-1} d \lambda \\
& =\frac{\left(\beta+\frac{1}{2} \sum_{k=1}^{N}\left(\ln x_{k}-\mu\right)^{2}\right)^{\frac{N}{2}+\alpha}}{\Gamma\left(\frac{N}{2}+\alpha\right)}\left[\frac{\Gamma\left(\frac{N}{2}+\alpha+1\right)}{\left(\beta+\frac{1}{2} \sum_{k=1}^{N}\left(\ln x_{k}-\mu\right)^{2}\right)^{\frac{N}{2}+\alpha+1}}\right] \\
& =\frac{\frac{N}{2}+\alpha}{\beta+\frac{1}{2} \sum_{k=1}^{N}\left(\ln x_{k}-\mu\right)^{2}} .
\end{aligned}
$$

From $E\left[\lambda \mid x_{1}, x_{2}, \ldots, x_{N}\right]=\hat{\lambda}$,

then $E\left[x_{1}, x_{2}, \ldots, x_{N} \mid \lambda\right]=e^{\mu+\frac{1}{2 \lambda}}$.

Therefore,

$$
E\left[x_{1}, x_{2}, \ldots, x_{N} \mid \lambda\right]=\exp \left[\mu+\frac{\beta+\frac{1}{2} \sum_{k=1}^{N}\left(\ln x_{k}-\mu\right)^{2}}{N+2 \alpha}\right] \text {. }
$$

The suitable premium that each policyholder pays must be proportional to both his/her number and size of the claims. The exact loss $x_{k}$ occurring from each claim results in different premiums for policyholders with the same number of claims. The Bayesian bonus-malus premium that must be paid fairly for all policyholders in the portfolio is equal to the product of the Bayesian premium based both on the frequency component in (5) and the severity component in (11) and can be expressed by

$$
\begin{aligned}
& \text { Premium }_{t+1}= \frac{(N+1)(N+2+t+\delta)}{(t+\delta)(N+1+t+\delta)} \\
& \cdot \exp \left(\mu+\frac{\beta+\frac{1}{2} \sum_{k=1}^{N}\left(\ln x_{k}-\mu\right)^{2}}{N+2 \alpha}\right) .
\end{aligned}
$$

To determine the bonus-malus premiums for a policyholder that must be paid according to the proposed model, we need to know the number of claims, age of the policy, claim amounts, and total claim amounts. All these values are generally shown in the portfolio.

The Bayesian bonus-malus premium at time $t=0$ is called the base premium. This is the initial premium that a new policyholder who joins the insurance must pay. Because of no claims at time $t=0$, in this study we considered $\ln x_{0}=\mu$. Therefore, the base premium can be expressed as

$$
\text { Premium }_{0}=\frac{(2+\delta)}{\delta(1+\delta)} \cdot \exp \left(\mu+\frac{\beta}{2 \alpha}\right) .
$$

\section{Numerical Application}

A data set was applied based on one-year automobile insurance policies taken out in 2004 or 2005 to calculate the model premiums introduced in this paper. This data set can be found on the website of the Faculty of Business and Economics, Macquarie University (Sydney, Australia), see also [19]. There were 67,856 policies in the total portfolio that at least one claim was 4,624 . There 
were also 4,333 policyholders who made claims once, 271 twice, 18 three times, and 2 at four times.

\subsection{BMS based on the Claim Frequency Component}

Claim frequency distribution was applied by the Poisson-Lindley distribution introduced in this article. The maximum likelihood estimator of the parameter of the Poisson-Lindley distribution was $\hat{\delta}=14.6238$, with Chi-Square goodness of fit test $\chi^{2}=2.1624$. We compared values of observed claim frequency between traditional PoissonExponential distribution and a Poisson-Lindley distribution. Results showed that the PoissonLindley distribution gave a better fit to the data than the Poisson-Exponential distribution. The expected claim frequencies were also compared as shown in Table 1.

Table 1. Observed frequency and expected frequency for estimated parameter values of Poisson-Exponential and Poisson-Lindley distributions

\begin{tabular}{crrr}
\hline \multirow{2}{*}{$\begin{array}{c}\text { Number } \\
\text { of claims }\end{array}$} & Observed & \multicolumn{2}{c}{ Expected frequency } \\
\cline { 3 - 4 } & frequency & Poisson-Exponential & Poisson-Lindley \\
\hline 0 & 63232 & 63253.85 & 63253.69 \\
1 & 4333 & 4290.03 & 4292.02 \\
2 & 271 & 290.96 & 290.30 \\
3 & 18 & 19.73 & 19.58 \\
4 & 2 & 1.34 & 1.32 \\
5 & 0 & 0.09 & 0.09 \\
\hline Total & 67856 & 67856 & 67856 \\
\hline Estimated parameter & 13.7444 & 14.6238 \\
& $\chi^{2}$ & 2.2866 & 2.1624 \\
& AIC & 36102.8938 & 36102.7548 \\
\hline
\end{tabular}

The Bayesian bonus-malus premiums based only on the frequency component were determined and calculated from (6), with results shown in Table 2 .

Table 2. Bonus-malus premiums based on the PoissonLindley distribution for the frequency component

\begin{tabular}{cccccc}
\hline \multirow{2}{*}{$t$} & \multicolumn{5}{c}{ Number of claims } \\
\cline { 2 - 6 } & 0 & 1 & 2 & 3 & 4 \\
\hline 0 & 100.00 & & & & \\
1 & 93.26 & 185.92 & 278.08 & 369.81 & 461.17 \\
2 & 87.37 & 174.23 & 260.67 & 346.74 & 432.50 \\
3 & 82.17 & 163.92 & 245.30 & 326.37 & 407.17 \\
4 & 77.56 & 154.75 & 231.63 & 308.24 & 384.61 \\
5 & 73.43 & 146.55 & 219.40 & 292.01 & 364.41 \\
6 & 69.72 & 139.18 & 208.39 & 277.39 & 346.21 \\
7 & 66.37 & 132.50 & 198.42 & 264.16 & 329.74 \\
\hline
\end{tabular}

From results in Table 2, a bonus with no claims in the first year represented $6.74 \%$ of the base premium. On the other hand, policyholders who made one claim during the first year must pay a malus at $85.92 \%$ of the base premium. Premiums decreased if the policyholders had a claim-free year and increased if claims occurred.

For comparative purposes, we computed the Bayesian bonus-malus premiums achieved under the traditional Poisson-Exponential model. The results are provided in Table 3.

Table 3. Bonus-malus premiums based on the PoissonExponential distribution for the frequency component

\begin{tabular}{cccccc}
\hline \multirow{2}{*}{$t$} & \multicolumn{5}{c}{ Number of claims } \\
\cline { 2 - 6 } & 0 & 1 & 2 & 3 & 4 \\
\hline 0 & 100.00 & & & & \\
1 & 93.22 & 186.44 & 279.65 & 372.87 & 466.09 \\
2 & 87.30 & 174.59 & 261.89 & 349.19 & 436.49 \\
3 & 82.08 & 164.17 & 246.25 & 328.33 & 410.42 \\
4 & 77.46 & 154.92 & 232.37 & 309.83 & 387.29 \\
5 & 73.33 & 146.65 & 219.98 & 293.30 & 366.63 \\
6 & 69.61 & 139.22 & 208.83 & 278.45 & 348.06 \\
7 & 66.26 & 132.51 & 198.77 & 265.02 & 331.28 \\
\hline
\end{tabular}

From the results in Table 3, an insured with a no claim in the first year is awarded a bonus of $6.78 \%$ on the base premium. On the other hand, an insured who makes one claim during the first year must pay a malus at $86.44 \%$ of the base premium.

We observed that the Bayesian bonus-malus premiums computing from the traditional PoissonExponential model are stricter with bad drivers than the proposed Poisson-Lindley model. Moreover, the premiums obtained from the Poisson-Exponential model are more generous with good drivers than the proposed Poisson-Lindley model.

\subsection{BMS based on Both Claim Frequency and Claim Severity Components}

We illustrated the premiums using the proposed model as in expression (12). For estimated parameters based on the severity component, we fitted the unconditional distribution as a LognormalGamma distribution in (7) to the claim sizes. Results gave maximum likelihood estimators for $\hat{\alpha}$ and $\hat{\beta}$ as 34.3312 and 274.9938 , respectively with $\mathrm{AIC}=82061.81$. We considered $\mu$ in the model in (12) and (13) as equal to 5.

Some symbols were defined for the purpose of convenience of explanation. We defined $\mathrm{NC}$ as the number of claims, CS as the claim size or severity of the claim, and TCS as the total claim size (Table 4-5).

We illustrated some cases where the amount of claim of an insured was different as shown in Table 6 in [20]. We considered the case of a policyholder who made four claims amounting to $235,471,706$, and 942. The results are shown in Table 4. 
Table 4. Bonus-malus premiums based on the PoissonLindley distribution for the frequency component and Lognormal-Gamma distribution for the severity component

\begin{tabular}{ccccccc}
\hline & NC & 0 & 1 & 2 & 3 & 4 \\
\cline { 2 - 7 }$t$ & CS & & 235 & 471 & 706 & 942 \\
& TCS & & 235 & 706 & 1412 & 2354 \\
\hline 0 & & 592.53 & & & & \\
1 & & 552.60 & 1041.67 & 1487.29 & 1905.13 & 2306.30 \\
2 & & 517.69 & 976.18 & 1394.19 & 1786.31 & 2162.93 \\
3 & & 486.90 & 918.40 & 1311.99 & 1681.35 & 2036.22 \\
4 & & 459.56 & 867.05 & 1238.89 & 1587.96 & 1923.43 \\
5 & & 435.12 & 821.10 & 1173.46 & 1504.34 & 1822.40 \\
6 & & 413.14 & 779.76 & 1114.56 & 1429.04 & 1731.40 \\
7 & & 393.26 & 742.37 & 1061.26 & 1360.88 & 1649.00 \\
\hline
\end{tabular}

Table 4 shows the premiums due for various claims when the age of the policy was up to seven years. Claim sizes were 235, 471, 706, and 942. The base premium, computed by (13), was equal to 592.53 , and this decreased with a claim-free year. On the other hand, if there was an accident with a claim size of 235 in the first year of observation, the policyholder will pay 1041.67. If a policyholder makes two claims in the first year, with the first claim of 235 and the second claim of 471, then he or she will pay 1487.29. Premiums increase as the number of claims increase. If in the second year the policyholder does not have an accident, then the premiums will reduce to $976.18,1394.19,1786.31$, and 2162.93 for numbers of claims from 1 to 4 , respectively. If the policyholder no longer has any claim, the premiums will again reduce for the third year and the fourth year, and so on.

For comparison purposes, we computed the Bayesian bonus-malus premiums again, which was achieved under the traditional Poisson-Exponential distribution for the frequency component and Lognormal-Gamma distribution for the severity component. The results are shown in Table 5.

Table 5. Bonus-malus premiums based on the PoissonExponential distribution for the frequency component and Lognormal-Gamma distribution for the severity component

\begin{tabular}{|c|c|c|c|c|c|c|}
\hline & $\mathrm{NC}$ & 0 & 1 & 2 & 3 & 4 \\
\hline \multirow[t]{2}{*}{$t$} & $\mathrm{CS}$ & & 235 & 471 & 706 & 942 \\
\hline & TCS & & 235 & 706 & 1412 & 2354 \\
\hline 0 & & 592.52 & & & & \\
\hline 1 & & 552.33 & 1044.53 & 1495.68 & 1920.87 & 2330.84 \\
\hline 2 & & 517.25 & 978.19 & 1400.68 & 1798.87 & 2182.80 \\
\hline 3 & & 486.36 & 919.77 & 1317.03 & 1691.43 & 2052.44 \\
\hline 4 & & 458.95 & 867.93 & 1242.81 & 1596.11 & 1936.77 \\
\hline 5 & & 434.47 & 821.63 & 1176.51 & 1510.96 & 1833.44 \\
\hline 6 & & 412.46 & 780.02 & 1116.92 & 1434.43 & 1740.59 \\
\hline 7 & & 392.58 & 742.41 & 1063.08 & 1365.29 & 1656.68 \\
\hline
\end{tabular}

From the results in Table 5, The base premium was equal to 592.52, and this decreased with a claim-free year. On the other hand, if there was an accident with a claim size of 235 in the first year of observation, the insured will pay 1044.53. If a policyholder makes two claims in the first year, with the first claim of 235 and the second claim of 471, then he or she will pay 1495.68. Premiums increase as the number of claims increase.

We observed that the base premiums and the premiums with a claim-free year are slightly different between bonus-malus premiums proposed in Table 4 and traditional bonus-malus premiums in Table 5. However, traditional bonus-malus premiums in Table 5 are stricter with bad drivers than the premiums presented in Table 4 when claims occurred.

\section{Conclusions}

A model was proposed to determine bonus-malus premiums. The model considered both claim frequency and claim severity components. Two mixing distributions as the Poisson with the Lindley distribution, and a mixed Lognormal with a Gamma distribution were investigated and employed in the model as frequency distribution and severity distribution, respectively. The premium was calculated by utilizing the Bayesian method.

We used an example of real automobile insurance data to illustrate our model. The fitted results of claim frequency were better than those of a traditional Poisson-Exponential model. Additionally, when claims occurred, bad drivers were liable to severe punishment under a traditional model. These may be problems with the insurer when the policyholder changes the company in the next year due to high premiums.

This proposed model is an alternative method for penalizing all policyholders in the portfolio. It is reasonable for punishing and rewarding both good and bad drivers. The obtained premiums could be useful for insurers in the competition in an insurance market.

To increase the impartiality for assigning the premium to all policyholders, one could consider distinguishing different types of claims. This could be a subject of further research.

\section{Acknowledgement:}

This research is financially supported by the Development and Promotion of Science and Technology Talents Project (DPST) of the Department of Mathematics, Faculty of Science, Khon Kaen University, Thailand. 
References:

[1] A. Espinal, R. Estrada, and C. Monsalve, Modelling TCP/IP Traffic of a Convergent Campus Wireless Network, International Journal of Circuits, Systems and Signal Processing, Vol.13, 2019, pp.611-616.

[2] S. Tu, Q. Lin, W. Wang, K. Sun, Y. Huang, and H.P. Nguyen, NCD-TAP: A Tracking Area Planning Approach Based on Newman Community Detection for HCN, International Journal of Circuits, Systems and Signal Processing, Vol.13, 2019, pp.140-147.

[3] L. Tremblay, Using the Poisson inverse Gaussian in bonus-malus systems, Astin Bulletin, Vol.22, No.1, 1992, pp.97-106.

[4] J. Lemaire, Bonus-malus systems in automobile insurance, Insurance: Mathematics and Economics, Vol.3, No.16, 1995, pp.277.

[5] J.F. Walhin and J. Paris, Using mixed Poisson processes in connection with bonus-malus systems, Astin Bulletin, Vol.29, 1999, pp.8199.

[6] I. Simeunovic, M. Balaba, and D. Bodroza, Pricing automobile insurance using mixed Poisson distributions, Industrija: časopis za ekonomiku industrije, Vol.46, No.1, 2018, pp. 61-78.

[7] S.E. Bulbul1 and K.B. Baykal1, Optimal Bonus Malus System Design in Motor Third Party Liability Insurance in Turkey: Negative Binomial Model, International Journal of Economics and Finance, Vol.8, No.8, 2016, pp.205-211.

[8] G. Tzougas, W.L. Hoon, and J.M. Lim, The Negative Binomial-Inverse Gaussian regression model with an application to insurance ratemaking. European Actuarial Journal, Vol.9, 2019, pp.323-344.

[9] N.E. Frangos and S.D. Vrontos, Design of optimal bonus-malus systems with a frequency and a severity component on an individual basis in automobile insurance, Astin Bulletin, Vol.31, No.1, 2001, pp.1-22.

[10] M. Mert and Y. Saykan, On a bonus-malus system where the claim frequency distribution is geometric and the claim severity distribution is Pareto, Hacettepe Journal of Mathematics and Statistics, Vol.34, 2005, pp.75-81.

[11] A. Ibiwoye, I.A. Adeleke, and S.A. Aduloju, Quest for optimal bonus-malus in automobile insurance in developing economies, $A n$ Actuarial Perspective. International Business Research, Vol.4, No.4, 2011, pp.74-83.

[12] W. Ni, C. Constantinescu, and A.A. Pantelous, Bonus-Malus systems with Weibull distributed claim severities, Annals of Actuarial Science, Vol.8, No.2, 2014, pp.217-233.

[13] A. Emad and I. Ali, Bayesian approach for bonus-malus systems with Gamma distributed claim severities in vehicles insurance, British Journal of Economics, Management, and Trade, Vol.14, No.1, 2016, pp.1-9.

[14] G. Tzougas, W. H. Yik, and M.W. Mustaqeem, Insurance ratemaking using the ExponentialLognormal regression model, Annals of Actuarial Science, Vol.14, 2019, pp.42-71.

[15] A. Jacob and Z. Wu, An Alternative Pricing System through Bayesian Estimates and Method of Moments in a Bonus-Malus Framework for the Ghanaian Auto Insurance Market, Journal of Risk and Financial Management, Vol.13, No.7, 2020, pp.143-157.

[16] A. Moumeesri and T. Talangtam, Transformed function based on Wang transform and Logtransform for insurance premium pricing, Proceeding of the International Conference on Applied Statistics 2016 (ICAS 2016), Thailand, 2016, pp.150-157.

[17] B.W. Mazviona and T. Chiduza. The use of statistical distributions to model claims in motor insurance, International Journal of Business, Economics and Law, Vol.3, Issue 1, 2013, pp.44-57.

[18] P. Sattayatham, and T. Talangtam, Fitting of finite mixture distributions to motor insurance claims, Journal of Mathematics and Statistics, Vol.8, No.1, 2012, pp.49-56.

[19] P. De Jong and G. Heller, Generalized Linear Models for Insurance Data. Cambridge University Press, 2008.

[20] E.G. Deniz, Bivariate credibility bonus malus premiums distinguishing between two types of claims. Insurance: Mathematics and Economics, Vol.70, 2016, pp.117-124.

\section{Creative Commons Attribution}

\section{License 4.0 (Attribution 4.0}

International , CC BY 4.0)

This article is published under the terms of the Creative Commons Attribution License 4.0 https://creativecommons.org/licenses/by/4.0/deed.en_US 